\title{
Effectiveness of Moringa Seed Powder, Scallop Powder and Other Commercial Powders in the Purification of Pond Water
}

\author{
Sharmin Zaman*1, Sabrina Sultana ${ }^{2}$, Anowara Begum², Marufa Zerin Akhter ${ }^{2}$ and Md. Latiful Bari ${ }^{1}$ \\ 1. Center for Advanced Research in Sciences, University of Dhaka, Dhaka-1000, Bangladesh \\ 2. Department of Microbiology, University of Dhaka, Dhaka-1000, Bangladesh \\ *Corresponding author's e-mail: sharmin_micro@yahoo.com
}

\begin{abstract}
Water turbidity is not a direct indicator of health risk; however, turbidity can provide food and shelter for pathogens as well as promote their re-growth in a distribution system and can interfere with the detection of bacteria and viruses in drinking water. In this study, moringa seed powder, ultra- $\mathrm{K}^{\circledR}$ powder, $\mathrm{FS}^{\circledR}$ powder and scallop powder were used individually or in combination to examine their antibacterial and coagulation properties in pond water. In addition to this treatment, attempts were also taken to convert treated water to drinking water through a 3 step natural bio-sand filtration process. This method would be very useful for areas of Bangladesh with scarcity of drinking water or areas prone to flooding. Results showed that Moringa seed powder alone has strong coagulant and antimicrobial effect at low doses. On the other hand, 0.01\% scallop powder has strong antimicrobial activity under typical environmental conditions. However, combination of these two powders showed effective coagulating and antimicrobial capacity to reduce the turbidity and inactivate the number of inherent microorganisms respectively, including coliform and E. coli within 5 min. Similar experimental findings were observed when the mixture of Moringa seed powder and sodium hypochlorite was used. On the other hand, both the commercial ultra-K and FS powder showed strong coagulant and antimicrobial effect within 1 min of application. When this treated water passed through natural bio-sand filtration (charcoal, stone and sand), the resulting water became potable. This small scale work was done in the laboratory and there is a need to scale-up this method to ascertain the reproducibility of the results. The present study suggests that Moringa seed powder and scallop powder are naturally available, cost-effective, and nontoxic antimicrobial agents that have potentials to convert pond water to drinkable water.
\end{abstract}

Key words: Pond water, Moringa seed powder, scallop powder, water purification. 